\title{
Thermal quenching of luminescence from buried and surface InGaAs self-assembled quantum dots with high sheet density
}

\author{
Z. F. Wei, S. J. Xu, ${ }^{\text {a) }}$ R. F. Duan, ${ }^{\text {b) }}$ Q. Li, and Jian Wang \\ Department of Physics and HKU-CAS Joint Laboratory on New Materials, The University of Hong Kong, \\ Pokfulam Road, Hong Kong, China \\ Y. P. Zeng \\ Institute of Semiconductors, Chinese Academy of Sciences, Beijing 100083, China \\ H. C. Liu \\ Institute for Microstructural Sciences, National Research Council, Ottawa K1A OR6, Canada
}

(Received 16 March 2005; accepted 7 September 2005; published online 20 October 2005)

\begin{abstract}
Variable-temperature photoluminescence (PL) spectra of Si-doped self-assembled InGaAs quantum dots (QDs) with and without GaAs cap layers were measured. Narrow and strong emission peak at $1075 \mathrm{~nm}$ and broad and weak peak at $1310 \mathrm{~nm}$ were observed for the buried and surface QDs at low temperature, respectively. As large as $210 \mathrm{meV}$ redshift of the PL peak of the surface QDs with respect to that of the buried QDs is mainly due to the change of the strain around QDs before and after growth of the GaAs cap layer. Using the developed localized-state luminescence model, we quantitatively calculate the temperature dependence of PL peaks and integrated intensities of the two samples. The results reveal that there exists a large difference in microscopic mechanisms of PL thermal quenching between two samples. () 2005 American Institute of Physics.

[DOI: $10.1063 / 1.2112176]$
\end{abstract}

\section{INTRODUCTION}

Since 1993, coherent semiconductor quantum dots (QDs) grown via strain-mediated self-organization method have attracted a growing interest due to their unique optoelectronic properties and great potential applications. ${ }^{1-5}$ While the buried QDs have been extensively investigated, very few studies have been devoted to the surface QDs without a cap layer. ${ }^{6-9}$ Available studies have commonly revealed that compared with the buried case, the surface QDs exhibit much weaker and broader emission peak with significantly lower energy. However, a detailed investigation on the luminescence mechanisms, especially on the thermal quenching mechanisms of luminescence of the surface QDs, is still lacking. We attempt to fill the void. Variable-temperature photoluminescence (PL) spectra from the buried and surface InGaAs QDs were measured in detail. A developed luminescence model was adopted to quantitatively reproduce the temperature dependence of the PL peak and intensity for both samples. The results revealed that there is a significant difference in the microscopic mechanisms of luminescence processes between the buried and surface cases.

\section{EXPERIMENT}

The $\mathrm{In}_{0.35} \mathrm{Ga}_{0.65} \mathrm{As}$ QD samples investigated in the present study were grown at $520^{\circ} \mathrm{C}$ on semi-insulating $\operatorname{GaAs}(001)$ substrates with EPI GEN II solid-state source molecular-beam epitaxy (MBE). Before the growth of 18

\footnotetext{
${ }^{\text {a) }}$ Author to whom correspondence should be addressed; electronic mail: sjxu @ hkucc.hku.hk

${ }^{b}$ Present address: Institute of Semiconductors, Chinese Academy of Sciences, Beijing 100083, China.
}

monolayer QD layer, a $200 \mathrm{~nm} \mathrm{GaAs}$ buffer layer was grown at $580{ }^{\circ} \mathrm{C}$. During the growth of a QD layer, Si atoms were simultaneously incorporated to make the dot layer doped with $\mathrm{Si}$ concentration of $4 \times 10^{17} \mathrm{~cm}^{-3}$. As mentioned earlier, the aim of the present work is to study the influence of GaAs cap layer on the optical properties of InGaAs QDs. For this purpose, two samples were grown and comparatively investigated. One is InGaAs QDs buried by a $100 \mathrm{~nm}$ GaAs cap layer grown at $580{ }^{\circ} \mathrm{C}$ while the other does not have such a cap layer.

For the variable-temperature PL measurements the samples were mounted on the cold finger of a Janis closed cycle cryostat with varying temperature range from 10 to $330 \mathrm{~K}$. The $632.8 \mathrm{~nm}$ line of a $\mathrm{He}-\mathrm{Ne}$ laser was employed as the excitation source. The luminescence signal was dispersed by an Acton SP300 monochromator and detected by a liquid-nitrogen-cooled Hamamatsu photomultiplier tube (PMT) detector whose detecting wavelength ranges from 300 to $1700 \mathrm{~nm}$. In order to obtain the dot information such as size and density, the atomic force microscopy (AFM) measurement of the surface QD sample was carried out with NanoScope IIIa AFM of Digital Instrument.

\section{RESULTS AND DISCUSSION}

Figure 1 shows the measured AFM image $\left(1 \times 1 \mu \mathrm{m}^{2}\right)$ of the surface QD sample. The sheet density of the dots is estimated from the image to be $7 \times 10^{10} \mathrm{~cm}^{-2}$. The average diameter and height of the dots are $\sim 46$ and $6 \mathrm{~nm}$, respectively. The $10 \mathrm{~K}$ PL spectra of the two samples are depicted in Fig. 2. It is clear that there is a big spectral distinction between the two samples. The buried QDs with a GaAs cap layer exhibit much stronger and narrower emission peak cen- 


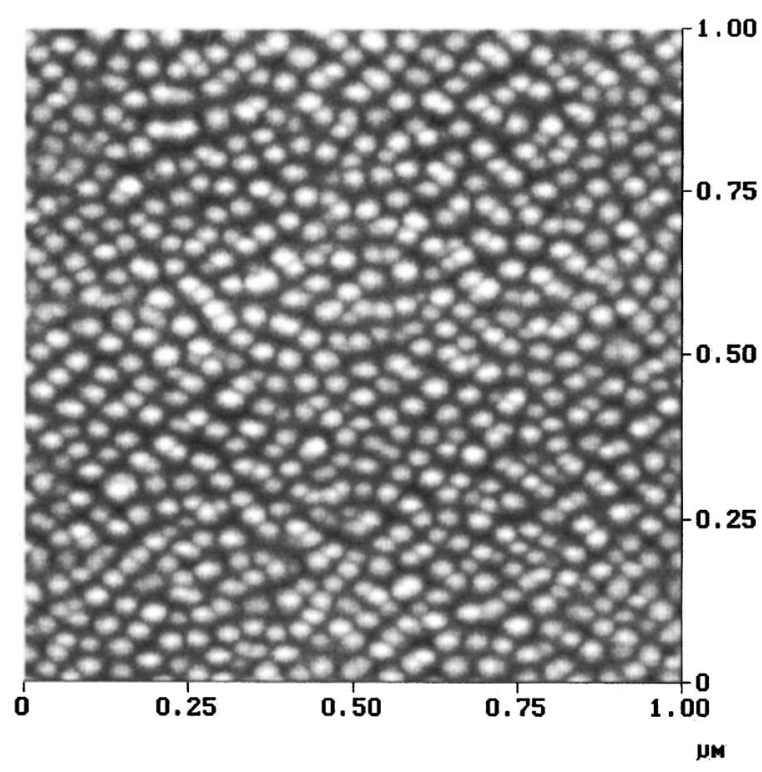

FIG. 1. Measured AFM image of the surface InGaAs QDs.

tered at $1.16 \mathrm{eV}$ while the surface QDs show weaker and broader peak at $0.95 \mathrm{eV}$. The linewidths of the PL peaks of the buried QDs and surface QDs are 50 and $110 \mathrm{meV}$, respectively. These spectral features are consistent with those reported in literature. ${ }^{6,7,9}$ Although the mechanisms are not well understood, the blueshift in the PL peak and the narrowing in the PL linewidth of the buried QDs may be attributed to the indium and gallium atom interdiffusion at the interfaces between InGaAs QDs and the surrounding GaAs matrix during the growth of GaAs cap layer at higher growth temperature. ${ }^{4,10}$ The atom interdiffusion can cause an increase of the gallium concentration in QDs and thus lead to the band-gap increase of the buried dots. On the other hand, it has been demonstrated that the atom interdiffusion can also result in strong modification of the residual strain inside the dots buried in the GaAs matrix. ${ }^{11}$ Simple estimation ${ }^{6}$ and finite element simulations ${ }^{4,9}$ show that the strain variation during the growth of GaAs cap layer for the buried QDs should be at least partially responsible for the observed blue-

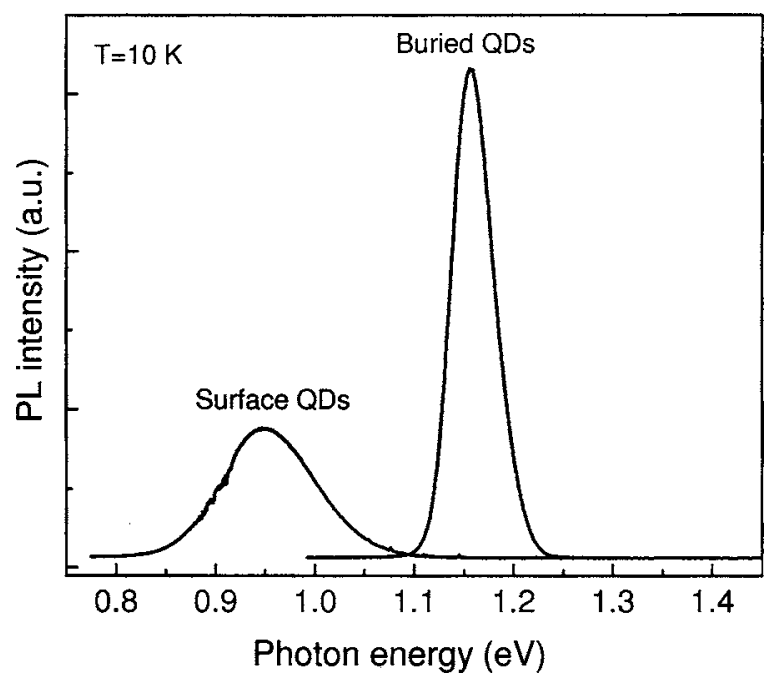

FIG. 2. Measured PL spectra of the surface and buried QDs at $10 \mathrm{~K}$.
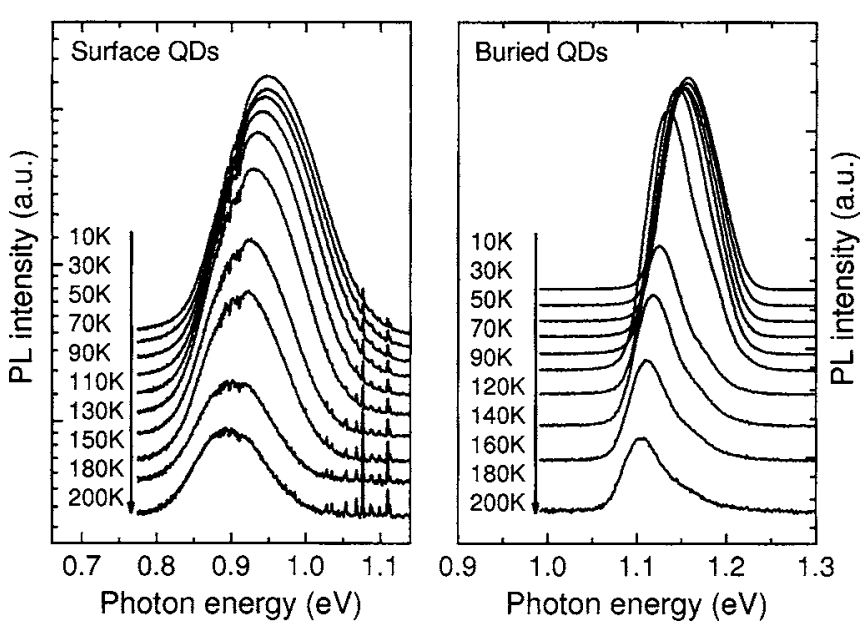

FIG. 3. Measured PL spectra of the surface and buried QDs at different temperatures.

shift in the PL peak. Following the work of Saito et al., ${ }^{6}$ we estimated the band-gap change of the $\operatorname{In}_{0.35} \mathrm{Ga}_{0.65}$ As QDs after growth of the GaAs cap layer using the strain-band offset model of the spherical dots developed by Nishi et al. ${ }^{12}$ It is found that the band-gap change is $\sim 201 \mathrm{meV}$ which is very close to the observed PL blueshift amount of $210 \mathrm{meV}$. This result indicates that the large shift in the PL peak of the buried QDs with respect to the surface QDs seems mainly due to the strain change before and after the growth of the GaAs cap layer. A more rigorous calculation based on the valence force field and the eight band $k \cdot p$ model also supports this assertion. ${ }^{13}$ Additionally, it is worth mentioning that the PL intensity of the surface QDs is much weaker than that of the buried QDs, as shown in Fig. 2. The surface states arising from the dangling bonds in the surface dots were proposed to be responsible for the weaker emission. ${ }^{6,9}$ Here we would like to point out that much weaker oscillator strength due to the wave functions' spatial separation of the ground electron and hole states in the surface dots should be at least one of the factors causing the weak luminescence. ${ }^{13}$

Figure 3 shows the PL spectra of both samples at different temperatures. As mentioned earlier, we are mainly concerned with the thermal quenching mechanisms of the emission peaks from the two samples. In order to make a more perspicuous comparison, the temperature dependence (solid squares) of PL peak positions and integrated intensities for the buried and surface dots is shown in Figs. 4 and 5, respectively. Note that the PL intensities were normalized. Similar to the spectral features discussed above, the temperature dependence of both the peak position and the integrated intensity is quite different for the two samples. In order to quantitatively interpret the temperature dependence of the quantities shown in Figs. 4 and 5, we adopted a luminescence model for localized-state ensemble recently developed by us. ${ }^{14,15}$ The major equations of the model are reproduced here. For PL peak position of many localized states, its temperature dependence is given by 

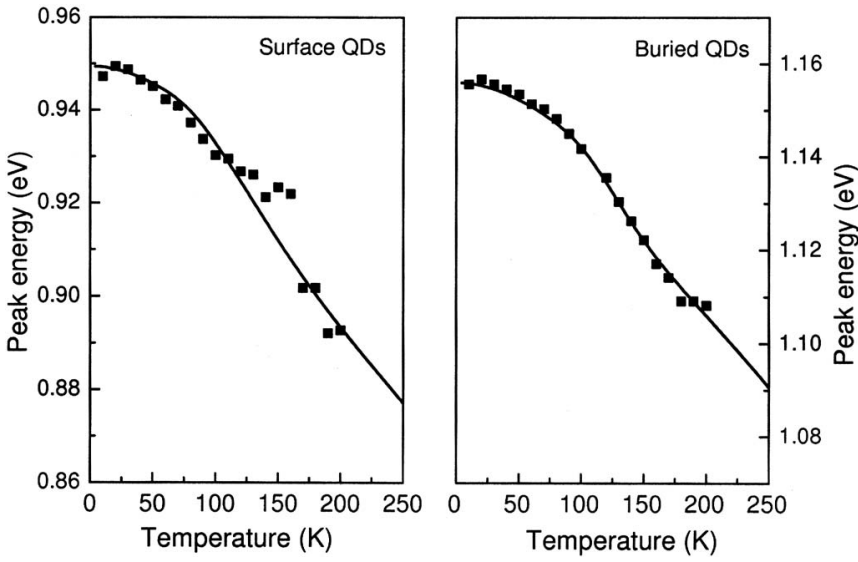

FIG. 4. Temperature dependence of the PL peak positions for the two samples. The solid squares are the experimental data while the solid lines represent the calculated results using the developed localized-state luminescence model.

$$
E(T)=E_{0}-\frac{\alpha T^{2}}{\Theta+T}-x \cdot k_{B} T
$$

where $E_{0}$ is the central energy of the localized-state distribution, which basically gives the PL peak position at $0 \mathrm{~K}$. The second term in the right-hand side of Eq. (1) is the wellknown Varshni empirical formula describing the temperature-induced band-gap redshift of an ideal semiconductor without localized states. In Varshni's empirical formula, $\alpha$ and $\Theta$ stand for the Varshni parameter and Debye temperature of the material, respectively. The third term in the right-hand side of Eq. (1) accounts for the abnormal temperature dependence of the PL peak position due to the thermal redistribution of carriers within the localized states under quasithermal equilibrium. The dimensionless variable $x(T)$ can be obtained by numerically solving the following equation:

$$
x e^{x}=\left[\left(\frac{\sigma}{k_{B} T}\right)^{2}-x\right]\left(\frac{\tau_{r}}{\tau_{\mathrm{tr}}}\right) e^{\left(E_{0}-E_{a}\right) / k_{B} T},
$$

where $\tau_{\text {tr }}$ is a time constant related to the carriers' thermal escape from the localized states, $\tau_{r}$ the recombination time of
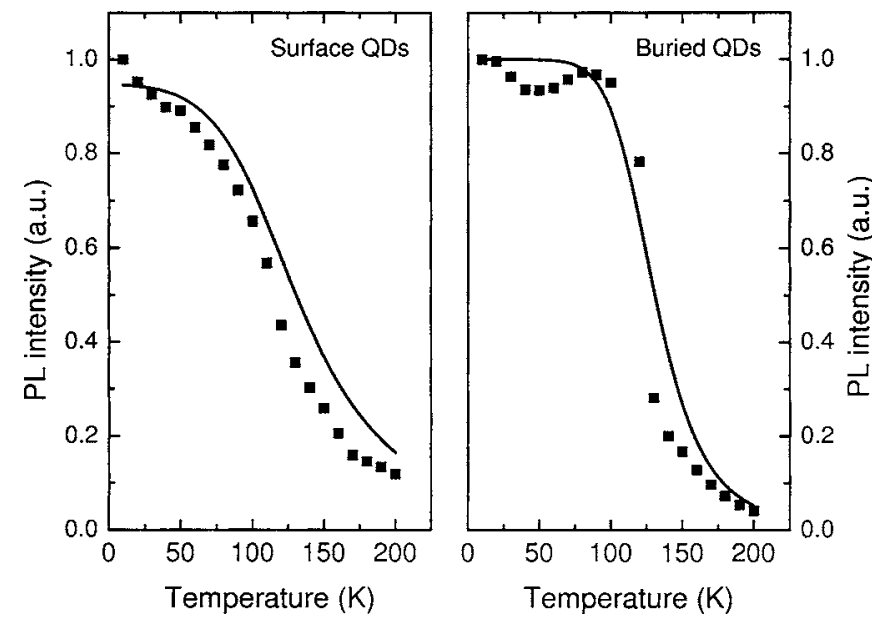

FIG. 5. The integrated intensities (normalized) of the emission peaks from the two samples vs temperature. The solid lines represent the calculated curves using the thermal transfer model. the localized carriers, $\sigma$ is a parameter characterizing the width of localized-state distribution, and $E_{a}$ represents the energetic position of a delocalized state to which the carriers occupying the localized states can be thermally activated at a rate of $\left(1 / \tau_{\mathrm{tr}}\right) \cdot e^{\left(E-E_{a}\right) / k_{B} T}$. For the integrated intensity of localized-state luminescence, its temperature dependence reads $^{15}$

$$
\begin{aligned}
& I(T) \propto\left(1+\left(1-\gamma_{c}\right)\right. \\
&\left.\times\left\{\exp \left[\frac{\left(E_{0}-E_{a}\right)+k_{B} T \ln \left(\tau_{r} / \tau_{\mathrm{tr}}\right)}{\sqrt{\left(k_{B} T\right)^{2}+2(\sigma / 2.41)^{2}}}\right]\right\}\right)^{-1},
\end{aligned}
$$

where $\gamma_{c}$ represents the recapture coefficient of the carriers already escaped by the localized states. This model has been successfully employed to quantitatively interpret the unusual temperature dependence of the PL peak from the localized carriers for different materials. ${ }^{14-17}$

The solid lines in Figs. 4 and 5 are the calculated results using Eqs. (1)-(3) when the following parameter values were taken for the buried and surface QDs. Buried case: $E_{0}$ $=1.156 \mathrm{eV}, \quad \sigma=13 \mathrm{meV}, \quad E_{a}-E_{0}=103 \mathrm{meV}, \quad$ and $\tau_{\mathrm{tr}} / \tau_{r}$ $=0.027 / 250$; surface case: $E_{0}=0.9495 \mathrm{eV}, \sigma=19 \mathrm{meV}, E_{a}$ $-E_{0}=65 \mathrm{meV}$, and $\tau_{\mathrm{tr}} / \tau_{r}=0.9 / 250$. For both cases, $\gamma_{c}$ is assumed to be zero and $\alpha=0.48 \mathrm{meV} / \mathrm{K}$ and $\Theta=270 \mathrm{~K}$ were adopted. From Figs. 4 and 5, it can be seen that the agreement between experiment and theory is good. It should be noted that the parameter values, in particular, $E_{a}-E_{0}$ and $\tau_{\text {tr }} / \tau_{r}$, are significantly different for the two cases. This reveals that the microscopic mechanisms of luminescence thermal quenching are quite different for the buried and surface QDs.

It is interesting to discuss more about the difference in thermal quenching mechanisms of the luminescence between the two samples. The magnitude of $E_{a}-E_{0}$ is, in fact, a measure of the so-called thermal activation energy which is a physical quantity frequently discussed in the luminescence thermal quenching in literature. It is easily proved that Eq. (3) can be reduced to a well-established model for two-level system $^{18}$ if the width of localized-state distribution is zero, i.e., $\sigma=0$. The larger is the value of $E_{a}-E_{0}$, the more difficult is the quenching of the PL intensity. Note that the value of $E_{a}-E_{0}$ is larger for the buried QDs. From Fig. 5, it can be seen that the PL intensity of the buried QDs remains nearly constant even if the temperature is increased up to $120 \mathrm{~K}$. This is a typical behavior theoretically expected ${ }^{19}$ and frequently observed for buried QDs. ${ }^{20}$ In contrast with the buried case, the PL intensity of the surface QDs rapidly drops with increasing temperature. If the surface states play a major role in the nonradiative decay of the carriers in the surface QDs, ${ }^{9}$ they should locate at $\sim 65 \mathrm{meV}$ above $E_{0}$. For the buried QDs, the thin wetting layer with higher energy can provide a main thermal escape path of carriers. ${ }^{20,21}$ Similarly, the energy of the wetting layer in the buried QDs studied at the present work is $103 \mathrm{meV}$ above $E_{0}$. In the thermal transfer model, another key parameter is $\tau_{r} / \tau_{\text {tr }}$ which always appears as a whole quantity. In the calculations, the same $\tau_{r}$ $=250 \mathrm{ps}$ was adopted ${ }^{15,20}$ while different values of $\tau_{\text {tr }}$ were taken for the buried and surface QDs. The physical origin 


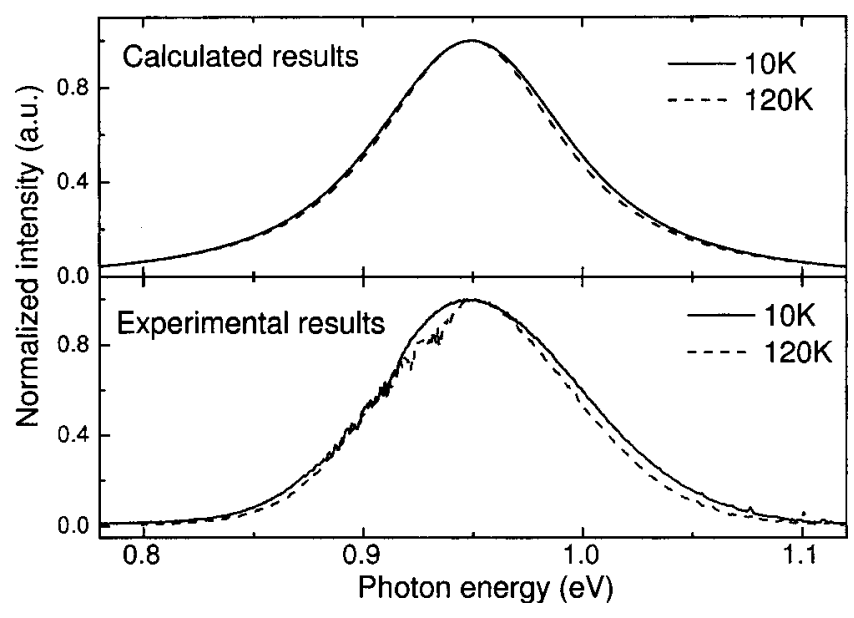

FIG. 6. Top: calculated distribution profiles of the localized carriers in the surface QDs at 10 and $120 \mathrm{~K}$; bottom: measured PL spectra of the surface QDs at 10 and $120 \mathrm{~K}$. The peak positions of calculated curves and measured PL spectra are manually coincided for clear comparison, respectively.

causing a much longer $\tau_{\text {tr }}$ in the case of the surface QDs is not clear. In spite of this fact, we can still explain why there is faster thermal quenching of the luminescence from the surface QDs. Note that the thermal escape rate of the carriers depends exponentially on the energy difference $E-E_{a}$, which is given by $\left(1 / \tau_{\mathrm{tr}}\right) \cdot e^{\left(E-E_{a}\right) / k_{B} T}$. In the both cases studied in the present work, $E-E_{a}$ is always negative. As is discussed earlier, there is a much smaller $E_{a}-E_{0}$ in the case of the surface QDs. The thermal escape rate of the localized carriers occupying the states in the energy range from $E_{0}$ to $E_{a}$ is thus much higher in the case of surface QDs, which mainly determines the thermal quenching of the localized-state luminescence. ${ }^{15}$ This may explain why the PL intensity of the surface QDs rapidly drops as the temperature increases.

According to the localized-state luminescence model, ${ }^{15}$ $N(E, T)$ represents the profile of localized-state distribution, which is essentially related with the line shape of the luminescence peak from the localized states. For given parameters shown earlier, $N(E, T)$ can be calculated at different temperatures. To learn more about the thermal quenching, a further discussion about the "shape" of the profile is given here. Taking the surface dot sample, for instance, depicted in Fig. 6 are the calculated curves at 10 and $120 \mathrm{~K}$, respectively. Note that the intensities are normalized and the peak positions for 10 and $120 \mathrm{~K}$ are manually coincided so as to make a more clear comparison. In our theoretical model, the thermal redistribution of carriers within localized state results in a shrinkage of PL linewidth in a certain temperature range. However, the PL peak asymmetrically shrinks with temperature, which can be observed from the calculated curves shown in Fig. 6. As the temperature increases, the low-energy side of the carrier distribution profile remains almost unchanged while its high-energy side shrinks much more obviously. This is due to the higher escaping rates of carriers occupying higher localized states with respect to the carriers occupying lower states. The measured PL spectra of the surface dots at same temperatures shown in the bottom figure in Fig. 6 are in good agreement with the theoretical prediction. These results show that the thermal quenching of the localized-state luminescence starts from the thermal escaping of the localized carriers occupying the higher electronic states. For the surface QDs, the thermal barrier for the localized carriers, for example, $E_{a}-E_{0}$, is relatively lower and thus its luminescence intensity is easier to quench.

\section{SUMMARY}

In summary, a comparative study on the luminescence thermal quenching in the surface and buried InGaAs QDs was conducted. The developed localized-state luminescence model was employed to quantitatively interpret the temperature dependence of the PL peak energy and intensity for both samples. It is concluded that the lower thermal activation barrier and higher thermal escape rate of carriers in the surface QDs are responsible for its faster luminescence thermal quenching.

\section{ACKNOWLEDGMENTS}

The work was supported by HK RGC CERG Grant under Contract No. HKU 7049/04P and HKU Research Grant under Contract No. 10204008.

${ }^{1}$ L. Jacak, P. Hawrylak, and A. Wójs, Quantum Dots (Springer, Berlin, 1998)

${ }^{2}$ D. Bimberg, M. Grundmann, and N. N. Ledentsov, Quantum Dot Heterostructures (Wiley, England, 1999).

${ }^{3}$ Semiconductor Quantum Dots, edited by Y. Masumoto and T. Takagahara (Springer, Berlin, 2002).

${ }^{4}$ J. Stangl, V. Holý, and G. Bauer, Rev. Mod. Phys. 76, 725 (2004).

${ }^{5}$ P. M. Petroff, Top. Appl. Phys. 90, 1 (2003).

${ }^{6}$ H. Saito, K. Nishi, and S. Sugou, Appl. Phys. Lett. 73, 2742 (1998).

${ }^{7}$ F. Ferdos, S. Wang, Y. Wei, A. Larsson, M. Sadeghi, and Q. X. Zhao, Appl. Phys. Lett. 81, 1195 (2002).

${ }^{8}$ C. F. Wang, A. Badolato, I. Wilson-Rae, P. M. Petroff, E. Hu, J. Urayama, and A. Imamoğlu, Appl. Phys. Lett. 85, 3423 (2004).

${ }^{9}$ Z. L. Miao, Y. W. Zhang, S. J. Chua, Y. H. Chye, P. Chen, and S. Tripathy, Appl. Phys. Lett. 86, 031914 (2005).

${ }^{10}$ S. J. Xu, X. C. Wang, S. J. Chua, C. H. Wang, W. J. Fan, J. Jiang, and X. G. Xie, Appl. Phys. Lett. 72, 3335 (1998).

${ }^{11}$ S. J. Xu, H. Wang, Q. Li, M. H. Xie, X. C. Wang, W. J. Fan, and S. L. Feng, Appl. Phys. Lett. 77, 2130 (2000).

${ }^{12}$ K. Nishi, A. Yamaguch, J. Ahopelto, A. Usui, and H. Sakaki, J. Appl. Phys. 76, 7437 (1994)

${ }^{13} \mathrm{H}$. B. Wu, S. J. Xu, and J. Wang (unpublished).

${ }^{14}$ Q. Li, S. J. Xu, W. C. Cheng, M. H. Xie, S. Y. Tong, C. M. Che, and H. Yang, Appl. Phys. Lett. 79, 1810 (2001).

${ }^{15}$ Q. Li, S. J. Xu, M. H. Xie, and S. Y. Tong, Europhys. Lett. 71, 994 (2005).

${ }^{16}$ J. S. Huang, X. D. Luo, X. D. Yang, Z. Sun, B. Q. Sun, Z. Y. Xu, and W. K. Ge, Chin. Phys. Lett. 21, 2529 (2004).

${ }^{17}$ S. J. Xu, Q. Li, J.-R. Dong, and S. J. Chua, Appl. Phys. Lett. 84, 2280 (2004).

${ }^{18}$ D. Curie, Luminescence in Crystals (Methuen, London, 1963), p. 206

${ }^{19}$ Y. Arakawa and H. Sakaki, Appl. Phys. Lett. 40, 939 (1982).

${ }^{20}$ Z. Y. Xu et al., Superlattices Microstruct. 23, 381 (1998).

${ }^{21}$ Z. Y. Xu et al., Phys. Rev. B 54, 11528 (1996). 